\title{
UNIFORM OPERATORS
}

BY

\author{
HSIAO - LAN WANG AND JOSEPH G. STAMPFLI ${ }^{1}$
}

\begin{abstract}
A general representation theorem for uniform operators is obtained which enables one to verify a conjecture of Cowen and Douglas in the presence of a mild additional restriction.
\end{abstract}

Let $\mathscr{B}(\mathscr{H})$ denote the algebra of bounded linear operators on a separable infinite-dimensional Hilbert space $\mathscr{H}$.

Definition. An operator $T \in \mathscr{B}(\mathscr{H})$ is uniform if $T$ is unitarily equivalent to $T \mid \mathscr{M}$ for every infinite-dimensional invariant subspace $\mathscr{M}$ of $T$.

EXAMPLES. 1. Let $\left\{e_{n}\right\}_{n=1}^{\infty}$ be an orthonormal basis for $\mathscr{H}$. Define the unilateral shift by setting $S e_{n}=e_{n+1}$ for $n=1,2, \ldots$ Then $S$ is uniform as may be easily seen from Beurling's Theorem [1].

2. Less interesting examples include a scalar multiple of the identity operator and transitive operators if any exist.

3. Let $\left\{e_{n}\right\}_{n=1}^{\infty}$ be an orthonormal basis for $\mathscr{H}$. Define the operator $D$ by

$$
D e_{1}=0, \quad D e_{n}=2^{-n} e_{n-1} \text { for } n>1
$$

Then $D$ (usually referred to as the Donoghue shift) has no infinite-dimensional invariant subspaces and, hence, is uniform. For more information on shifts of this type see [ 3 or 5 ].

More examples and information about uniform operators can be found in [8]. In particular, it is proved there that all uniform operators possess a cyclic vector.

In [2] Cowen and Douglas ask whether every uniform operator is a function of the unilateral shift, that is, an analytic Toeplitz operator. (They do not use the term "uniform" and are careful to exclude transitive operators.) We observe that, while Example 3 supplies an example of a uniform operator which is not a function of the unilateral shift, the answer to the Cowen-Douglas question is yes, if we impose a mild restriction.

We now obtain a representation theorem for uniform operators which possess a proper infinite-dimensional invariant subspace. This result will enable us to answer a number of questions. In particular, it tells us that any uniform operator with

Received by the editors January 20, 1983 and, in revised form, August 24, 1983 and February 22, 1984. 1980 Mathematics Subject Classification. Primary 47B35, 47B37.

Key words and phrases. Uniform operator, cyclic vector, invariant subspace, Toeplitz operator.

${ }^{1}$ The second author gratefully acknowledges the support of the National Science Foundation. 
nonempty compression spectrum is unitarily equivalent to an analytic Toeplitz operator.

Note well. From this point on we assume $T \neq \lambda I$.

Lemma 2. Let $T \in \mathscr{B}(\mathscr{H})$ be uniform and assume $T$ has an infinite-dimensional invariant subspace $\mathscr{M}$. Then $T$ is unitarily equivalent to an operator of the form

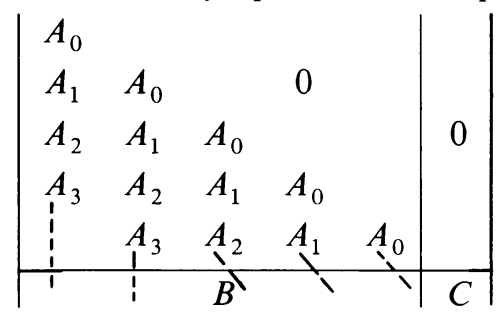

The terms $B, C$ may be nonexistent; i.e. they may or may not appear.

Proof. Let $\mathscr{H}_{0}=\mathscr{H}$ and $\mathscr{H}_{1}=\mathscr{M}$. Let $T_{0}=T \mid \mathscr{H}$ and $T \mid \mathscr{H}_{1}=T_{1}$. Since $T_{0}$ is uniform it follows that there exists a unitary operator $V: \mathscr{H}_{0} \rightarrow \mathscr{H}_{1}$ such that $T_{1} V=V T_{0}$. (Unitary here means isometric onto.) Note that $V \mathscr{H}_{0}=\mathscr{H}_{1}$ and $\operatorname{dim}\left[\mathscr{H}_{0} \ominus \mathscr{H}_{1}\right]$ may be finite or infinite but not 0 . Let $\mathscr{H}_{n}=V^{n} \mathscr{H}_{0}$ for $n=1,2, \ldots$ Since $V$ is an isometry $\mathscr{H}_{n} \neq\{0\}$. Let $\mathscr{L}=\mathscr{H}_{0} \ominus \mathscr{H}_{1}$. Since $V \mathscr{L} \subset \mathscr{H}_{1}$ it follows that $V \mathscr{L} \perp \mathscr{L}$. Thus $V^{n+1} \mathscr{L} \perp V^{n} \mathscr{L}$ for $n=0,1, \ldots$ since $V$ is unitary. Clearly $V^{n} \mathscr{L} \subset \mathscr{H}_{n}$ $=V^{n} \mathscr{H}_{0}$.

Claim. $\mathscr{H}_{n} \ominus \mathscr{H}_{n+1}=V^{n} \mathscr{L}$.

Let $g \in \mathscr{H}_{n+1}$. Then $g=V^{n+1} h$. For any $l \in \mathscr{L}$ we see that $\left(V^{n} l, g\right)=$ $\left(V^{n} l, V^{n+1} h\right)=(l, V h)=0$ since $l \perp V \mathscr{H}$. Thus $V^{n} \mathscr{L} \in \mathscr{H}_{n} \ominus \mathscr{H}_{n+1}$. The opposite inclusion follows in the same way. Denote $V^{n} \mathscr{L}$ by $\mathscr{L}_{n}$ for $n=0,1, \ldots$. Clearly $\mathscr{L}_{n} \perp \mathscr{L}_{n+1}$ for $n=0,1, \ldots$ Let $\mathscr{E}_{1}=\oplus_{0}^{\infty} \mathscr{L}_{n}$ and $\mathscr{E}_{2}=\mathscr{H} \oplus \mathscr{E}_{1}$. Then $\mathscr{H}=\mathscr{E}_{1} \oplus \mathscr{E}_{2}$, where $\mathscr{E}_{1}=\mathscr{L} \oplus V \mathscr{L} \oplus \cdots, V$ is isometric on $\mathscr{E}_{1}, V \mathscr{E}_{2}=\mathscr{E}_{2}$ and $V$ is unitary on $\mathscr{E}_{2}$. What we have written is just the Wold decomposition for $V$. It is well known that $\mathscr{E}_{2}=\bigcap_{1}^{\infty} V^{n} \mathscr{H}_{0}$.

Claim. $\mathscr{H}_{n}$ is invariant under $T$.

Denote $T \mid \mathscr{H}_{n}$ by $T_{n}$ for $n=1,2, \ldots$ and assume we have shown that $T \mathscr{H}_{k} \subset \mathscr{H}_{k}$ for $k=1, \ldots, n$. Note that $k=1$ is just our initial hypothesis $T \mathscr{H}_{1} \subset \mathscr{H}_{1}$. Thus $T \mathscr{H}_{n+1}=T V \mathscr{H}_{n}=T_{1} V \mathscr{H}_{n}=V T \mathscr{H}_{n} \subset V \mathscr{H}_{n}=\mathscr{H}_{n+1}$, which establishes the claim. Since $V T=T_{1} V$ and $T \mathscr{H}_{n} \subset \mathscr{H}_{n}$, it follows that $V T_{k}=T_{k+1} V$ for $k=1,2, \ldots$ by simply restricting the set of vectors to which we apply the operators. By induction we see that $V^{k} T_{0}=T_{k} V^{k}$ for $k=1,2, \ldots$

Claim. Let $P_{k}$ be the projection of $\mathscr{H}_{0}$ on $\mathscr{L}_{k}$. Then $P_{k+n} T P_{m+n}$ is unitarily equivalent to $P_{k} T P_{m}$ for $n=1,2, \ldots$ Observe that $P_{k} T P_{m}=0$ for $k<m$ since $\mathscr{H}_{n}$ is invariant under $T$. We assign a basis $\left\{e_{j}^{(0)}\right\}$ to $\mathscr{L}=\mathscr{L}_{0}$. Let $\left\{e_{j}^{(n)}=V^{n} e_{j}^{(0)}\right\}$ be a basis for $\mathscr{L}_{n}$. Fix $k, m, j, q$ where $k \geqslant m$. Then

$$
\begin{aligned}
\left(T e_{j}^{(m)}, e_{q}^{(k)}\right) & =\left(T_{m} e_{j}^{(m)}, e_{q}^{(k)}\right)=\left(T_{m} V^{m} e_{j}^{(0)}, V^{m} e_{q}^{(k-m)}\right) \\
& =\left(V^{* m} T_{m} V^{m} e_{j}^{(0)}, e_{q}^{(k-m)}\right)=\left(T_{0} e_{j}^{(0)}, e_{q}^{(k-m)}\right) .
\end{aligned}
$$

Since the value of the last expression depends only on the difference $k-m$, we have established the claim. 
If we define $P_{k} T P_{m}: \mathscr{L}_{m} \rightarrow \mathscr{L}_{k}$ to be $A_{k-m}$ then $T$ clearly has the form

$$
\left|\begin{array}{ccc|c}
A_{0} & & & \\
A_{1} & A_{0} & 0 & \\
1 & A_{1} & A_{0} & 0 \\
\hline & B & \ddots
\end{array}\right|
$$

on $\oplus_{k=0}^{\infty} \mathscr{L}_{k} \oplus \mathscr{E}_{2}$.

We next wish to consider the possible dimensions for $\mathscr{E}_{2}$. While $\mathscr{E}_{2}=\{0\}$ is possible (take $T$ to be the shift and $\mathscr{H}_{1}=\mathscr{M}$ to equal $z \mathscr{H}^{2}$ ), we can rule out some occurrences.

LEMMA 3. $\mathscr{E}_{2}$ cannot be finite dimensional.

Proof. Note that

$$
\mathscr{E}_{2}=\bigcap_{n=0}^{\infty} V^{n} \mathscr{H}_{0}=\left[\bigoplus_{n=0}^{\infty} V^{n} \mathscr{L}\right] .
$$

Clearly $\mathscr{E}_{2}$ is invariant under $T$. If $h \in \mathscr{E}_{2}$ then $h \perp V^{n} \mathscr{L}$ for $n=0,1, \ldots$ and conversely. Recall that $V \mathscr{E}_{2}=\mathscr{E}_{2}$. Assume $\mathscr{E}_{2}$ is finite dimensional.

Claim. $\mathscr{E}_{2}$ reduces $T$. Assume $0 \neq\left(T_{0} e_{j}^{(m)}, f\right)$ for some $f \in \mathscr{E}_{2}$ and fixed $m, j$. Recall that

$$
V^{k} T_{0}=T_{k} V^{h} \quad \text { for } k=1,2, \ldots
$$

Thus

$$
\begin{aligned}
0 & \neq \alpha=\left(T_{0} e_{j}^{(m)}, f\right)=\left(V^{k *} T_{k} V^{k} e_{j}^{(m)}, f\right) \\
& =\left(T_{k} e_{j}^{(k+m)}, V^{k} f\right)=\left(T_{k} e_{j}^{(k+m)}, f_{k}\right) .
\end{aligned}
$$

Thus there exists a O.N. sequence $g_{k} \in \mathscr{E}_{1}$ such that $\left\|P_{\mathscr{E}_{2}} T g_{k}\right\| \geqslant \alpha \geqslant 0$. But the unit ball of $\mathscr{E}_{2}$ is compact and

$$
\lim _{k \rightarrow \infty}\left(T g_{k}, f\right)=\lim _{k \rightarrow \infty}\left(g_{k}, T^{*} f\right)=0
$$

for all $f \in \mathscr{E}_{2}$, which is a contradiction. Thus $\mathscr{E}_{2}$ reduces $T$. Since $T \mid \mathscr{E}_{2}$ must have an eigenvalue, say $\lambda_{0}$, then $T \mid \mathscr{E}_{1}$ has the same eigenvalue $\lambda_{0}$ and, by repetition, $\lambda_{0}$ is an eigenvalue of infinite multiplicity. In that case $T=\lambda_{0} I$, which is impossible.

LeMMA 4. If $\operatorname{dim} \mathscr{L}<\infty$ then $\mathscr{E}_{2}=\{0\}$.

Proof. Assume $\mathscr{E}_{2} \neq\{0\}$. By the previous lemma, $\mathscr{E}_{2}$ must be an infinite-dimensional invariant subspace of $T$. Since $T \mid \mathscr{E}_{2} \cong T$ it follows that $T$ must have the form

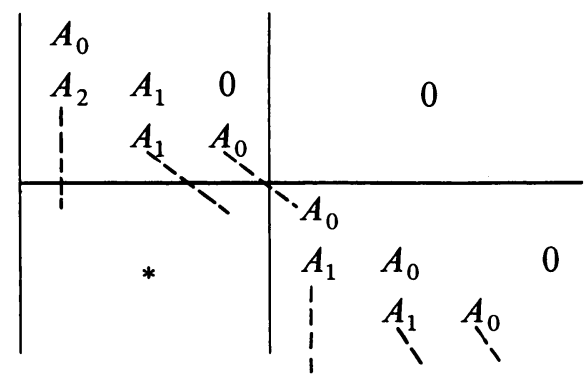


If $U T=\left(T \mid \mathscr{E}_{2}\right) U$, denote $U \mathscr{L}$ by $\mathscr{M}$. (This $\mathscr{M}$ plays the same role in the lower right corner of the $2 \times 2$ matrix as $\mathscr{L}$ did in the upper left corner.) Since $T$ is uniform and, hence, has a cyclic vector it follows that $1 \geqslant \operatorname{dim} \operatorname{ker} T^{*} \geqslant \operatorname{dim} \operatorname{ker} A_{0}^{*}$. Let $A_{\mathscr{M}}$ denote $P_{\mathscr{M}} A_{0} \mid \mathscr{M}$. Then there exists a vector $f \in \mathscr{M}$ and a $\lambda_{0}$ such that

$$
\left(\left(A_{\mathscr{M}}-\lambda_{0}\right) g, f\right)=0 \quad \text { for all } g \in \mathscr{M} \text {. }
$$

But by the remark above, Range $\left(A_{\mathscr{M}}-\lambda_{0}\right)^{\perp}=\{f\}$. Recall that $V T_{0}=T_{1} V$. Then for any $g \in \mathscr{E}_{2}$ it follows that

$$
0=\left(\left(T_{1}-\lambda_{0}\right) V g, f\right)=\left(V\left(T_{0}-\lambda_{0}\right) g, f\right)=\left(\left(T_{0}-\lambda_{0}\right) g, V^{*} f\right) .
$$

Since Range $\left(T_{0}-\lambda_{0}\right) \mid \mathscr{E}_{2}$ has codimension 1 it follows that $V^{*} f=\bar{\alpha} f$, whence $V f=\alpha f$. Having established the last relation we will show that $T^{*} f=A_{\mathscr{M}} f=\bar{\lambda}_{0} f$. Let $e_{j}^{(m)} \in \mathscr{L}_{m}$ for arbitrary $m, j$. Then

$$
\begin{aligned}
\left|\left(T e_{j}^{(m)}, f\right)\right| & =\left|\left(V^{* k} T_{k} V^{k} e_{j}^{(m)}, f\right)\right|=\left|\left(T_{k} e_{j}^{(m+k)}, V^{k} j\right)\right| \\
& =\left|\left(T e_{j}^{(m+k)}, \alpha^{k} f\right)\right|=\left|\left(e_{j}^{(m+k)}, T^{*} f\right)\right| .
\end{aligned}
$$

But the last term tends to zero since $\left\{e_{j}^{(m+k)}\right\}_{k=1}^{\infty}$ is orthonormal. Thus $\left|\left(e_{j}^{(m)}, T^{*} f\right)\right|$ $=0$ for all $m, j$. Hence $T^{*} f=A_{\mathscr{M}} f=\bar{\lambda}_{0} f$. But this implies $\operatorname{dim} \operatorname{ker}\left(T^{*}-\lambda\right) \geqslant 2$, which is impossible since $T$ is uniform.

Definition. An operator $T \in B(\mathscr{H})$ is an analytic Toeplitz operator if there exists an $f \in H^{\infty}$ such that $T=f(S)$, where $S$ is the unilateral shift. Equivalently, there exists an orthonormal basis $\left\{e_{n}\right\}_{n=0}^{\infty}$ for $\mathscr{H}$ such that $\left(T e_{i}, e_{j}\right)=\left(T e_{i+k}, e_{j+k}\right)$ for all $i, j, k=0,1,2, \ldots$ and $\left(T e_{i}, e_{j}\right)=0$ for $j<i$.

THEOREM 5. Let $T \in B(\mathscr{H})$ be uniform. Let $\mathscr{M}$ be an invariant subspace of $T$ of finite codimension. Then $T$ is an analytic Toeplitz operator.

Proof. Since $\mathscr{L}=\mathscr{H} \ominus \mathscr{M}$ is finite dimensional it follows from Lemma 4 that $E_{2}=\{0\}$. Thus

$$
T=\left|\begin{array}{lll}
A_{0} & & \\
A_{1} & A_{Q}-A_{0} & 0
\end{array}\right| \text { on } \bigoplus_{k=0}^{\infty} V^{k} \mathscr{L} .
$$

Since $\operatorname{dim} \mathscr{L}<\infty$, clearly $A_{0}^{*}$ and, hence $T^{*}$, has an eigenvector, say $f \neq 0$. Thus $R=\{f\}^{\perp}$ is an invariant subspace of $T$ of codimension one. Set $\mathscr{L}_{1}=\{f\}$. Then

$$
T=\left|\begin{array}{ccc}
a_{0} & & \\
a_{1} & a_{0} & 0 \\
1 & a_{1} & a_{0}
\end{array}\right| \text { on } \bigoplus_{k=0}^{\infty} V_{1}^{k} \mathscr{L}_{1},
$$

where the $a_{j}$ 's are scalars. Thus $T$ is an analytic Toeplitz operator.

Corollary 6. Let $T$ be uniform. If $\operatorname{ker}(T-\lambda)^{*} \neq\{0\}$ for some $\lambda \in \mathscr{C}$, then $T$ is an analytic Toeplitz operator. 
REMARK. It is possible for $T$ to have the form of Lemma 1 with $\mathscr{L}$ finite but not one dimensional. For example the shift operator satisfies all conditions with $\mathscr{L}=$ $\left\{e_{1}, e_{2}\right\}$ and

for $j \geqslant 2$.

$$
A_{0}=\left|\begin{array}{ll}
0 & 0 \\
1 & 0
\end{array}\right|, \quad A_{1}=\left|\begin{array}{ll}
0 & 1 \\
0 & 0
\end{array}\right| \quad \text { and } \quad A_{j}=\left|\begin{array}{ll}
0 & 0 \\
0 & 0
\end{array}\right|
$$

It is easy to see that an analytic Toeplitz operator is uniform if it has the same invariant subspace lattice as the unilateral shift. Sarason $[7,6]$ showed that the analytic Toeplitz operator $T_{f}$ with $f \in H^{\infty}$ has the same invariant subspaces as the unilateral shift if and only if the polynomials in $f$ are weak-star dense in $H^{\infty}$. But it is not known whether every uniform analytic Toeplitz operator has the same invariant subspaces as the unilateral shift or not.

Question. If $f \in H^{\infty}$ and $T_{f}$ is uniform, is $f$ a weak-star generator of $H^{\infty}$; i.e., are the polynomials in $f$ weak-star dense in $H^{\infty}$ ?

LEMMA 7. Let $T \in B(\mathscr{M})$ be uniform. Let $\mathscr{M}$ be an infinite-dimensional subspace of $T$ when $\operatorname{dim}(\mathscr{H} \in \mathscr{M})$ is finite. Then $T$ has a matrix representation with blocks continuing out to some countable ordinal.

The form will be described in more detail below.

Proof. By Lemma 2, $T$ has the form

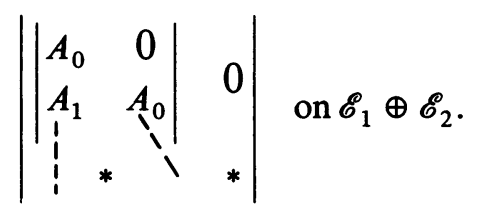

If $\mathscr{E}_{2} \neq\{0\}$ then $\mathscr{E}_{2}$ is an infinite-dimensional invariant subspace for $T$ and, hence, $T \mid \mathscr{E}_{2} \cong T$.

Let

$$
B_{0}=\left|\begin{array}{ccc}
A_{0} & & \\
A_{1} & A_{0} & 0 \\
\vdots & A_{1} & \underbrace{A_{0}}_{0}
\end{array}\right| .
$$

Then repeating the previous argument with $\mathscr{M}$ replaced by $\mathscr{E}_{2}$, we see that $T$ has the form

If $\mathscr{E}_{3}=\{0\}$, stop. If not, set

$$
|| \begin{array}{ccc}
B_{0} & & 0 \\
B_{1} & B_{0} & \\
1 & B_{1} & B_{0} \\
1 & \vdots & 0 \\
1 & * &
\end{array} \mid \text { on } \mathscr{E}_{2}^{\perp} \oplus \mathscr{E}_{3} .
$$

$$
C_{0}=\left|\begin{array}{lll}
B_{0} & & 0 \\
B_{1} & B_{0} & \\
1 & B_{1} & B_{0}
\end{array}\right| .
$$


Then $T$ has the form

$$
|| \begin{array}{ccc}
C_{0} & & 0 \\
C_{1} & C_{0} & \\
1 & C_{1} & C_{0} \\
1 & \vdots & 0 \\
1 & * & *
\end{array} \mid \quad \text { on } \mathscr{E}_{4}^{\perp} \oplus \mathscr{E}_{4} .
$$

If $\mathscr{E}_{4}=\{0\}$, stop. If $\mathscr{E}_{4} \neq\{0\}$, set $D_{0}$ equal to the upper left block and continue.

The process may terminate at any stage or may continue out to $\boldsymbol{\aleph}_{0}$. At a limit ordinal we can begin all over again and proceed by transfinite induction.

The procedure should be clear by now. If the " $\mathscr{E}_{2}$ " space in Lemma 2 is $\{0\}$, stop. If not, form the new blocks by the rule illustrated above. Since our underlying space $\mathscr{H}$ is separable and we pick up a new vector orthogonal to earlier ones at each step, the process must terminate before we reach the first uncountable ordinal.

Question. Do there exist uniform operators where the representation continues out to a preassigned ordinal? We have been unable to find a uniform operator where $\mathscr{E}_{2} \neq\{0\}$. In the case of the unilateral shift one can choose $\mathscr{L}$ to be infinite dimensional, but it is not hard to see that $\mathscr{E}_{2}=\{0\}$.

LEMma 8. Let $T$ be uniform. Let $R$ be the closed linear span of the finite-dimensional invariant subspace of $T$. Then $R$ is finite dimensional or $R=\mathscr{H}$.

Proof. Assume $R$ is infinite-dimensional. Then $T \cong T \mid R$. Since the finite-dimensional invariant subspaces of $(T \mid R)$ span the underlying space $R$, the same must be true for $T$. Hence $R=\mathscr{H}$.

TheOREM 9. Let $T \in B(\mathscr{H})$ be uniform and assume $T$ has an infinite-dimensional invariant subspace $\mathscr{M} \neq \mathscr{H}$. Then $T$ has no finite-dimensional invariant subspaces.

Proof. Assume $T$ has a finite-dimensional invariant subspace. Then, in particular, $T$ has an eigenvalue $\zeta$ and the subspace $F_{\zeta}=\{x \in \mathscr{H}: T x=\zeta x\}$ is nonempty. Since $T \neq \lambda I$, it follows that $F_{\zeta}$ is finite dimensional. Apply Lemma 2 to $T$ and $\mathscr{M}$ and let $V$ be the unitary operator which satisfies $T_{1} V=V T_{0}$. Then $V F_{\zeta} \subset F_{\zeta}$ and, since $F_{\zeta}$ is finite dimensional, $V F_{\zeta}=F_{\zeta}$. Hence $F_{\zeta} \subset \cap_{k=1}^{\infty} V^{k} \mathscr{H}=\mathscr{E}_{2}$. As a first step we split $\mathscr{H}$ as $\mathscr{E}_{1} \oplus \mathscr{E}_{2}$ and find $\mathscr{E}_{2} \supset F_{\zeta} \neq\{0\}$. Continuing the process as in Lemma 7, we obtain $\mathscr{E}_{3}$, and the argument just given will show $F_{\zeta} \subset \mathscr{E}_{3}$.

Follow the transfinite induction scheme described in Lemma 7. For every $E_{j}$ where $j=1,2, \ldots$ we see that $E_{j} \supset F_{\zeta}$ and, moreover, $E_{\omega} \supset F_{\zeta}$ even for the limit ordinals $\omega$. However, $\mathscr{H}$ is separable and each $E_{\beta}$ contains a vector $f_{\beta} \neq 0$, where $f_{\beta} \perp E_{\alpha}$ for $\alpha<\beta$. Thus we must eventually arrive at an ordinal $\tau$ where $E_{\tau}=\{0\}$. Since $E_{\tau} \supset F_{\zeta}$, this is a contradiction.

\section{BIBLIOGRAPHY}

1. A. Beurling, On two problems concerning linear transformations in Hilbert space, Acta Math. 81 (1949), 239-255.

2. M. J. Cowen and R. G. Douglas, On moduli for invariant subspaces, Invariant Subspaces and Other Topics (Timisoara/Herculane, 1981), Operator Theory: Advanced Applications, 6, Birkhauser, Basel, 1882, pp. $65-73$. 
3. W. F. Donahue, The lattice of invariant subspaces of a completely continuous quasi-nilpotent transformation, Pacific J. Math. 7 (1957), 1031-1035.

4. R. G. Douglas, Structure theory of operators. I, J. Reine Angew. Math. 232 (1968), 180-193.

5. N. K. Nikol'skii, The unicellularity and nonunicellularity of weighted shift operators, Dokl. Akad. Nauk SSSR 172 (1967), no. 2; English transl., Soviet Math. Dokl. 8 (1967), 91-94.

6. D. Sarason, Invariant subspaces and unstarred algebra, Pacific J. Math. 17 (1966), 511-518.

7. __ Weak-star density of polynomials, J. Reine Angew. Math. 252 (1972), 1-15.

8. H. Wang, On uniform operators, Thesis, Indiana Univ., 1982.

9. H. Wold, $A$ study in the analysis of stationary time series, Almqvist \& Wiksell, Stockholm, 1938.

DEPARTMENT OF MATHEMATICS, INDIANA UNIVERSITY, BLOOMINGTON, INDIANA 47405 